\title{
Sepsis-Triggering Pathogen Determines the Bacterial Translocation Profile and the Clinical Outcome
}

\author{
Ana Maria Alvim Liberatore ${ }^{1 *}$, Rosa Maria Silva ${ }^{2}$ and Ivan Hong Jun \\ Koh $^{1}$ \\ ${ }^{1}$ Laboratório de Pesquisa Experimental - Universidade Federal de São Paulo, Brazil \\ ${ }^{2}$ Department of Microbiology, Parasitology and Immunology, Universidade Federal \\ De São Paulo, Brazil \\ *Corresponding Author: Ana Maria Alvim Liberatore, Laboratório de Pesquisa \\ Experimental - Universidade Federal de São Paulo, Brazil.
}

DOI: $10.31080 /$ ASMI.2020.03.0511
Received: January 30, 2020

Published: February 08, 2020

(C) All rights are reserved by Ana Maria Alvim

Liberatore., et al.

\begin{abstract}
Introduction: Sepsis is a systemic infection associated with organ dysfunction. The so-called "gut hypothesis" of the Multiple Organ Dysfunction Syndrome holds that the gut may stimulate or exacerbate the host state of inflammatory response either by gut tissue hypoxia-ischemia injury or by intestinal barrier dysfunction followed by bacterial translocation (BT). BT has been associated with the worsening of the inflammatory status in experimental sepsis. In this study, we examined the kinetic changes of the aerobic and anaerobic facultative Gram-negative bacteria from gut microbiota and its relation to the BT following the Gram-positive and Gramnegative sepsis-induction in rats.

Methods: Wistar-EPM rats, weighing 200 - 250g were submitted to E. coli or S. aureus sepsis $\left(10^{8} \mathrm{CFU} / \mathrm{mL}\right.$, iv. $)$ and, after 6, 12 and $24 \mathrm{hs}$, intestinal segments, feces and mesenteric lymph nodes were removed and cultured to determine the number of aerobe and anaerobe facultative Gram-negative microorganisms. Sham group (saline injection) and Naïve animals were used as controls.

Results: The data showed the occurrence of an expressive bacterial overgrowth in small bowel since $6 \mathrm{hs}$ and from $12 \mathrm{hs}$ in the large bowel following sepsis challenge. These data suggest that the overgrowth in sepsis occurs acutely and, in a crania-caudal manner throughout the intestine. BT events also occurred acutely (6h) and at a higher rate in sepsis induced by E. coli, as compared to $S$. aureus (12hs) sepsis. Mortality was observed only in E. coli-sepsis. Control Sham group showed a low grade and a transient overgrowth, demonstrating that even minor surgical trauma can interfere with the gut microbiota equilibrium.

Conclusion: The significant mortality (60\%) associated with the BT positivity in E.coli-sepsis points out possible participation of BT in the exacerbation of the pre-existing systemic inflammatory state in sepsis which was dependent on the sepsis-inducing bacterial strain. Furthermore, the increase of fecal bacterial overgrowth and BT event occurred concurrently suggesting that monitoring Gram-negative fecal overgrowth may predict the BT event.
\end{abstract}

Keywords: Sepsis; Bacterial Translocation; Gut Microbiota; Gut Bacterial Overgrowth; Experimental Sepsis

\section{Abbreviations}

BT: Bacterial Translocation; PRRs: Pattern-Recognition Receptors; PAMPs: Pathogen-Associated Molecular Patterns; TLRs: TollLike Receptors; MODS: Multiple Organ Dysfunction Syndrome;
GALT: Gut-Associated Lymphoid Tissue; MLN: Mesenteric Lymph Nodes; SIRS: Systemic Inflammatory Response Syndrome; MAMPs: Microbe-Associated Molecular Patterns; ExPEC: Extra-Intestinal Pathogenic E. coli. 


\section{Introduction}

According to the consensus published in 2016, sepsis should be defined as life-threatening organ dysfunction caused by a deregulated host response to infection [1]. The severe systemic infection with multiple systems impairments is still beyond the current pathophysiology comprehension. Likewise, the sepsis treatment is neither target-specific nor effective, and the acute and chronic outcomes are still associated with high mortality worldwide $[2,3]$.

Some of the most frequently isolated bacteria in sepsis patients are Staphylococcus aureus, Escherichia coli, Klebsiella spp., and Pseudomonas aeruginosa [4]. The host innate immune cells recognize several bacterial virulence factors via pattern-recognition receptors (PRRs). Pathogens like Gram-negative and Gram-positive bacteria and their products can activate different specific inflammatory pathways by the so-called pathogen-associated molecular patterns (PAMPs) and recognized by toll-like receptors (TLRs) [5].

The detection of microorganisms and/or their products by the immune system in sepsis generates a cascade of inflammatory events that demand prompt adaptations in the therapeutic target, based on the state of the commitment of the biological damages and that still are being elucidated.

During the aggravation phases of sepsis and shock, the splanchnic blood flow is partially diverted to preserve the vital organ's blood flow, thus resulting in splanchnic organ hypoxia-ischemia. This splanchnic hypo-flux has been related to increased intestinal mucosal permeability, the intestinal cytopathic phenomena, and further dysfunction [6-7].

In this context, the so-called "gut hypothesis" of the Multiple Organ Dysfunction Syndrome (MODS) holds that the gut immune activation may stimulate or exacerbate the host inflammation state either by gut tissue hypoxia-ischemia injuries or by intestinal barrier dysfunction and subsequent bacterial translocation. These events would induce the gut-associated lymphoid tissue (GALT) activation, and further release of biologically active factors through the lymph to the systemic bloodstream, amplifying the host systemic inflammatory response [8-11].

The term bacterial translocation (TB), described by Berg and Garlington (1979), refers to the passage of viable bacteria residing in the gastrointestinal tract, and/or its products, to the extra-intes- tinal sites [12]. Three conditions are described for its occurrence: the overgrowth of intestinal bacteria, intestinal barrier dysfunction, and the immunodeficiency of the host [13]. The intestinal hypothesis of MODS is based on the BT as a possible cause of systemic infection and/or subsequent aggravation of inflammatory state of the host [8,14-15]. 0'Boyle., et al. [16] suggested that alterations in the intestinal barrier function are unproductive of the enteric BT, showing that in sepsis and other critical illness conditions, BT event is probably dependent on several associated factors. Taken into account that the study of BT is complex because it cannot be performed noninvasively, the studies that evaluate the pathophysiology of this event are mostly experimental.

Experimental studies have enrolled BT in the exacerbation of the inflammatory response. In this sense, the literature has shown that BT can induce the release of activated inflammatory products through the mesenteric lymphatic duct [10,17-19], even in the absence of bacteria in the efferent lymph [19]. In addition, a systemic inflammatory state was observed in naive rats submitted to an injection of mesenteric lymph collected from septic rats. Besides, in previous experiments, the induction of BT by the confinement of a high E.coli concentration $\left(10 \mathrm{~mL}\right.$ of $\left.10^{10} \mathrm{CFU} / \mathrm{mL}\right)$ in the small intestine in septic rats resulted in a significant increase in the mortality rate. These experiments in rats showed that BT phenomena combined with systemic inflammatory state of sepsis may worsen the sepsis outcomes [19-20]. However, the spontaneous luminal bacterial overgrowth during the sepsis, with subsequent induction of the BT, resulting in the worsening of the clinical state of sepsis has not been demonstrated yet in experimental or clinical settings.

Although it is known, that pathogens and their products activate distinct pathways of the inflammatory response and immune response [21] it is not known whether different pathogens with distinct pathways of host inflammatory activation may promote variations in bacterial translocation rate and consequently a different activation of GALT and clinical outcome in sepsis.

Considering the relevance of BT and subsequent GALT activation in the exacerbation of the inflammatory response and organ dysfunction, this study aimed to investigate if sepsis induced by Gram-positive and Gram-negative bacteria promotes distinct changes in Gram-negative intestinal microbiota of rats and in BT rates. In addition, this study investigated the induction and profile of translocation and its correlation to sepsis clinical outcomes. 


\section{Materials and Methods}

Animals

Female Wistar-EPM rats of three months of age, weighing 200 to 250 grams, were obtained from the University Animal Colony (CEDEME). Three animals were allocated per cage and fed with standard rat chow and tap water ad libitum until the experiments. Following seven days of adaptation, animals were submitted to experiments. The study was approved by the Federal University of São Paulo -UNIFESP Ethical Committee (CEP 099504).

\section{Bacterial strains for sepsis induction}

The Escherichia coli R-6 (ONT:H2 serotype) [20] was used to induce Gram-negative (G-) sepsis, and S. aureus (ATCC 29213) was used to induce Gram-positive $(\mathrm{G}+)$ sepsis. The bacteria were suspended in saline solution to a final concentration of $10^{8} \mathrm{CFU} / \mathrm{mL}$ and added formalin solution $(0.5 \%)$ to obtain non-viable bacterium inoculum. After 48 hours, the formalin solution was removed by successive washing with sterile saline, and the non-viable bacteria were used as the sepsis-inoculum. Inoculum of dead bacteria was used in this sepsis model to prevent its recovery in mesenteric lymph node culture and to overlap with translocated Gramnegative bacteria from the gut. A previous study [20] showed that sepsis challenge with either live or non-viable bacteria prompted similar clinical outcomes.

\section{Experimental groups}

Sepsis groups (SG): animals inoculated intravenously (iv) with $2 \mathrm{~mL}$ of $10^{8} \mathrm{CFU} / \mathrm{mL}$ Gram-negative bacteria (S8G-) or with Gram-positive bacteria (S8G+). ( $n=6 /$ group/period); Sham group (Sham): animals submitted to $2 \mathrm{~mL}$ of saline solution injection. ( $\mathrm{n}$ =6/period); Naive group (Naive): without any procedure $(n=6)$.

\section{Procedures}

Initially, a kinetic study of the fecal bacteria concentration was carried out in six rats submitted to sepsis by E. coli (S8) in order to determine the time required for the fecal microbiota to change. Fecal samples were collected for microbiological procedures before $(\mathrm{T}=0)$ and after sepsis induction ( $\mathrm{T}=6 \mathrm{hrs}, \mathrm{T}=12 \mathrm{hrs}, \mathrm{T}=24 \mathrm{hrs}$, $\mathrm{T}=48 \mathrm{hrs}, \mathrm{T}=72 \mathrm{hrs}, \mathrm{T}=5$ days, and $\mathrm{T}=9$ days). Based on fecal bacterial count and overgrowth findings, the periods for monitoring intestinal microorganisms and BT in sepsis were considered appropriate at 6,12 and 24 hours after sepsis challenge.
Sepsis induction and sample collection

General anesthesia was induced by an intramuscular injection of $0.1 \mathrm{~mL} / 100 \mathrm{~g}$ (bodyweight) of Ketamine and Hydroxychloral (4:1). After identification, isolation, and cannulation of the jugular vein, sepsis was induced by intravenous injection of $2 \mathrm{~mL}$ of inocu$\operatorname{lum}\left(10^{8} \mathrm{CFU} / \mathrm{mL}\right.$ of $E$. coli R-6 or $S$. aureus, $\mathrm{n}=6 /$ group/period). Sham group received $2 \mathrm{~mL}$ of saline injection ( $\mathrm{n}=6 /$ period). At 6,12 , and 24 hours periods, the intestinal segments (duodenum, jejunum, ileum, and cecum), feces and mesenteric lymph nodes (MLN) were removed through median laparotomy under the same general anesthesia. Samples from the Naive group were collected using the same process. All surgical procedures were carried out using aseptic and antiseptic techniques. After sample collection, the animals were euthanized by the section of the aorta still under general anesthesia.

\section{Microbiological procedures}

Intestinal segments and MLN samples were weighed, macerated, diluted in phosphate-buffered saline, filtered, and then quantitatively cultured onto MacConkey agar (Difco-USA) in aerobic incubation at $37{ }^{\circ} \mathrm{C}$ for $24-48$ hours to permit the growth of aerobe and facultative anaerobe Gram-negative microorganisms. Fecal samples were weighed, serially diluted in sterile saline solution and cloth-filtered to eliminate particulate material before been cultured in the same conditions. Isolated bacteria from MLN, intestinal segments and feces were identified by colonial characteristics and by using biochemical tests EPM-Mili medium (Probac-Brazil) and Simon's Citrate medium (Difco-USA). The colonies were counted, and the results expressed in $\log _{10}$.

\section{Mortality analysis}

The investigation of the mortality index was carried out in other separate groups of rats after sepsis induction by the same inoculum of E.coli and S. aureus (n = 5/group/bacteria). Animals were monitored per 30 days for mortality.

\section{Statistics}

Bacteria recovered from all samples were expressed as the median of $\log _{10}$. Statistical analysis was performed using Friedman Test to analyze differences between results in the same group, and Kruskal Wallis and Mann Whitney test to compare the data between different groups. Differences were considered significant when the P-value was less than $0.05(\mathrm{p}<0.05)$. 


\section{Results and Discussion}

\section{Fecal kinetic study}

Initially, the fecal microbiological kinetic changes following Gram-negative sepsis (E. coli) were compared to Sham group findings to evaluate the period of bacterial overgrowth detectable in feces and thus guide the time length of overgrowth study in the gut.

The results showed that in both groups there was a rapid increase in the fecal concentration of Gram-negative bacteria that returned to similar amounts to the basal (T0) concentration in $48 \mathrm{hrs}$ for the Sham group and $72 \mathrm{hrs}$ in the S8G- sepsis group. After 12 hours the S8G- group presented bacterial counts significantly higher than T0 (before sepsis induction), reaching a maximum after $24 \mathrm{hrs}$. These results showed that a single sepsis challenge was able to induce a fast and expressive overgrowth of the gut indigenous Gram-negative bacteria. The same analysis in the Sham group showed that even a very low-grade surgical trauma under general anesthesia was able to promote a rapid and significant increase in bacterial overgrowth during the first 12 hours. However, its return towards the basal levels ( $\mathrm{T}=0$ ) was faster $(48 \mathrm{hs})$ as compared to the sepsis group that remained altered for a more prolonged period (72hs) (Figure 1).

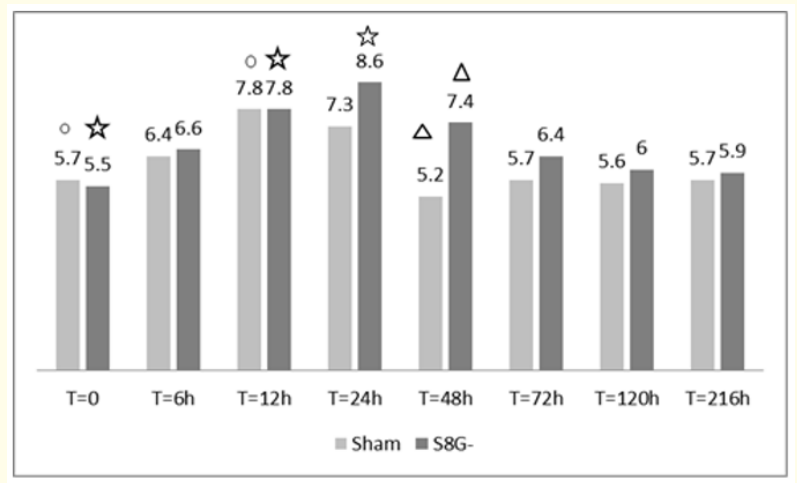

Figure 1: Kinetics of the fecal bacterial recovery following Gramnegative sepsis (S8G-) vs. Sham groups. Data were expressed in the mean of $\log 10$ counts/g of feces; $p<0,05$ between the periods

of sepsis (star), between periods of Sham (circle); between groups (triangle).

\section{Bacterial recovery from intestinal segments and feces}

In accordance with feces results, three study periods $(6,12$ and 24 hours) were chosen to investigate the alterations of intestinal bacterial counts in the small and large bowel compartments after the sepsis induction. Sepsis was performed using two different bacterial species, Gram-negative E.coli (S8G-) and Gram-positive $S$. aureus (S8G+) to compare the results. The Sham group was performed to evaluate the influence of surgical trauma on the gut aerobic and facultative anaerobic Gram-negative bacterial counts. Naïve animals, without any procedure, were used as negative controls.

The microbiological findings showed a bacterial overgrowth with onset in the small bowel of rats at six hours after sepsis induction only in S8G- sepsis group, significant when compared to the Naïve group. On the other hand, the large bowel bacterial counts were unaltered at this initial period (Figure 2). At 12 hours after sepsis, a significant increase of bacterial counts was observed in both small and large bowel segments in both S8G- and S8G+ groups, as compared to control groups. At this period, the large intestine compartment showed the highest counts, mainly in the S8G- sepsis group. Significant results were observed between S8G- and S8G+ groups (Figure 3). At 24 hours period, the cecum and feces of both sepsis groups showed high bacterial counts, more significantly in S8G- group than in S8G+. At this period, the duodenum and jejunum bacterial counts of all groups returned to similar levels to control groups, suggesting that bacterial overgrowth was a transitory phenomenon in these compartments in this sepsis model (Figure 4). The standard deviation indicated fluctuations in bacterial concentration mainly in the small intestine in sepsis groups, as compared to the Naive group. Sham groups showed bacterial concentrations lower than sepsis groups at all examined periods.

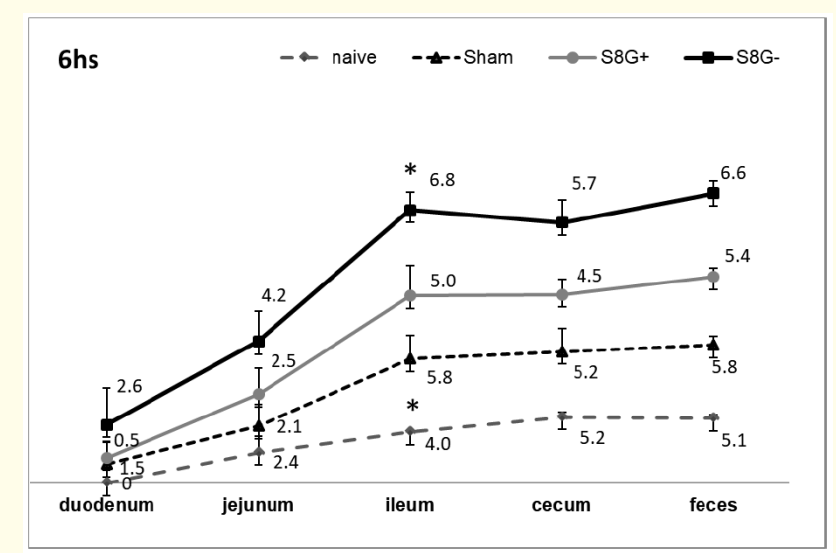

Figure 2: Bacterial recovery from intestinal segments and feces at 6 hs after S8G+ and S8G- sepsis induction. Data were expressed as mean of $\log 10$ counts/g with standard deviation: $\mathrm{p}<0,05$ Sepsis vs Naïve (*). 


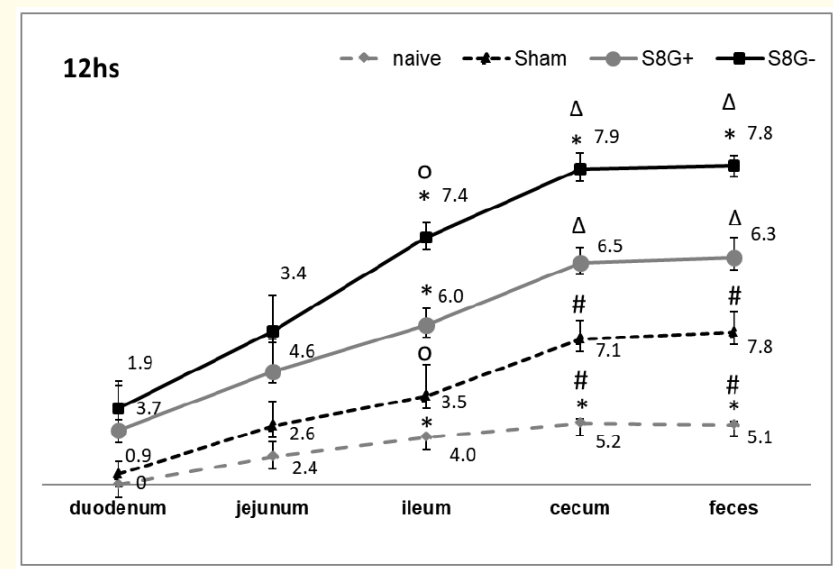

Figure 3: Bacterial recovery from intestinal segments and feces at $12 \mathrm{hs}$ after S8G+ and S8G- sepsis induction. Data were expressed as mean of $\log 10$ counts/g with standard deviation: $\mathrm{p}<0,05$ Sepsis vs Naïve (*); Sham vs Naive (\#);Sepsis vs Sham (o); S8G- vs S8G+ $(\Delta)$

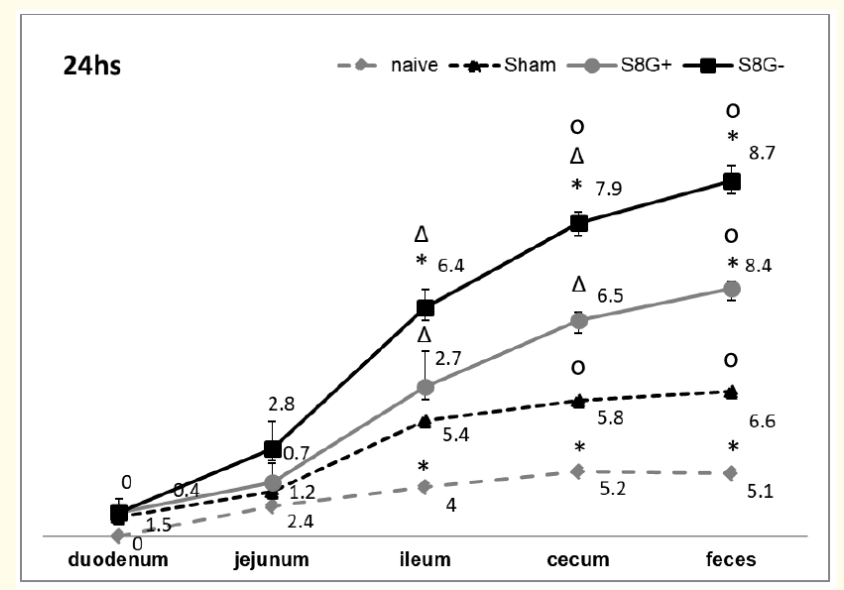

Figure 4: Bacterial recovery from intestinal segments and feces at 24hs after S8G+ and S8G- sepsis induction. Data were expressed as mean of $\log 10$ counts/g with standard deviation: $\mathrm{p}<0,05$ Sepsis vs Naïve (*); Sepsis vs Sham (o); S8G- vs S8G+ $(\Delta)$.

The results showed a significant and fast bacterial overgrowth of the aerobic and facultative anaerobic Gram-negative intestinal bacteria, befalling from the upper to the lower gastrointestinal tract along the periods. These results showed that a single sepsis challenge with E. coli or S. aureus could cause intestinal microbiota imbalance, although the sepsis caused by E. coli has promoted a higher overgrowth when compared to $S$. aureus, in all studied periods.

These findings have shown that sepsis may result in distinct clinical consequences depending on the pathogenic characteristics of the bacterial strain involved, the virulence factors and their products, microbe-associated molecular patterns (MAMPs), molecular interactions between the microorganism and the host, tolllike receptors, pattern recognition receptors (PRRs), and the host immune responsiveness mechanism [5].

In a recent study with $74 \mathrm{E}$. coli strains, isolated from 74 hospitalized patients, it was indicated a significant variability of profiles of virulence factors, being $61 \%$ extra-intestinal pathogenic $E$. coli (ExPEC) with intrinsic virulent potential, and 39\% with a low virulence capacity. These findings demonstrated the importance of discriminating the E. coli isolates relative to their virulence mechanisms involved in extraintestinal E. coli infections [22].

Alterations in the intrinsic intestinal control factors, such as intestinal secretions, luminal $\mathrm{pH}$, and the phasic propulsive motor activity, that occur in sepsis and other critical diseases, may have favored the gut bacterial overgrowth [23]. A clinical study in patients with Systemic Inflammatory Response Syndrome (SIRS) also showed a gut dysbiosis, however demonstrating the decrease of the obligatory anaerobic bacteria in feces [24].

\section{Bacterial translocation}

In the same context, the question to be raised is about the importance of the overgrowth event related to the BT mechanism, in health and in critical disease.

The spontaneous BT to the MLN was observed in $67 \%$ of animals at the period of six hours post S8G- sepsis challenge and remained positive with a similar index in the subsequent periods. The BT mean in S8G- was $61 \%$ to all periods, in contrast to $17 \%$ in the S8G+ group (Table 1). Control groups, Sham and Naïve, showed BT negative.

These findings may be related to the absence of bacterial overgrowth in intestinal segments of the S8G+ group at six and $24 \mathrm{hrs}$ in the ileum, an intestinal site related to the BT occurrence [19]. On the contrary, a persistent overgrowth in the luminal contents up 
to 24hrs in S8G- group might correlate with the persistence of BT (Table 1). These results show, once again, that bacterial virulence and its consequences differ between microorganisms.

\begin{tabular}{|c|cc|}
\hline $\begin{array}{c}\text { Time after } \\
\text { Sepsis } \\
\text { induction }\end{array}$ & $\begin{array}{c}\text { E. coli } \\
\text { S8G- }\end{array}$ & $\begin{array}{c}\text { S. aureus } \\
\text { S8G+ }\end{array}$ \\
\hline $6 \mathrm{~h}$ & $67 \%$ & $0 \%$ \\
\hline $12 \mathrm{~h}$ & $50 \%$ & $50 \%$ \\
\hline $24 \mathrm{~h}$ & $67 \%$ & $0 \%$ \\
\hline Mean $\%$ & $61 \%$ & $17 \%$ \\
\hline
\end{tabular}

Table 1: Percentage of animals with positive BT per period.

In previous studies, the confinement of an exogenous $E$. coli with concentrations $\left(10^{10} \mathrm{CFU}\right)$ in the small intestine, a higher concentration of bacteria than is usual for rats $\left(10^{5-6} \mathrm{CFU}\right)$, has translocate within two hours to MLN, liver, and spleen in healthy animals, showing the efficiency of the small intestine barrier has limitations even in healthy animals and can be supplanted in the bacterial overgrowth condition [19].

Interestingly, in both sepsis groups, only gut origin E. coli was able to translocate to MLNs among other luminal Gram-negatives, S8G- (mean $2.6+0,32 \log _{10}$ ) and S8G+ (mean $2.4+0,41 \log _{10}$ ). This fact suggests that $E$. coli ought to possess some specific mechanisms that facilitate its translocation and/or permanence to extra-intestinal sites based on their virulence factors, survival strategies, resistance to complement, or own mechanisms that ease to bacterial invasion. Besides that, this bacterium is considered as the bacteria that most translocate to extra-intestinal sites [25].

There are several factors related to the clinical evolution of sepsis that may contribute to the weakening of the intestinal defense components, namely: gut epithelial damage, exacerbated inflammatory response or immune dysfunction, hypovolemic shock, splanchnic hypoperfusion with intestinal cytopathic hypoxia, endothelial damage, increased pro-inflammatory and nitric oxide mediators [11,19-20,26,27-28]. In this sense and under these conditions, microbial overgrowth in the intestinal tract with a wide surface of communication with the internal milieu may have had a very favorable condition to propitiate the rapid occurrence of nonphysiological BT as early as in six hours of sepsis.
The mechanisms involved in BT are difficult to evaluate in clinical settings, as they require invasive procedures for their better understanding and, in this sense, experimental studies can bring better contributions to the understanding of its pathophysiology.

\section{Mortality}

Sepsis mortality was $60 \%$ in S8G- and $0 \%$ in the S8G+ group, although the same bacterial concentration was used. The mortality rate in S8G- was similar to BT index (61\%), suggesting a possible relationship between BT and mortality in Gram-negative sepsis group. The sepsis-induced by $S$. aureus didn't promote an expressive BT index either mortality.

These data suggest that the consequences of sepsis were dependent on the virulence mechanisms of the bacterial strain that causes sepsis, besides interactive factors involving bacteria and the host.

Further pathophysiologic studies are necessary to observe if increasing the $S$. aureus concentration will produce a similar outcome to E. coli obtained in this present study.

The gut is considered by some authors as the "Motor" of MODS due to its important role in the exacerbation of the inflammatory events in sepsis and other critical illnesses [6-8,15,18]. The GALT activation by BT and the release of biologically active factors via lymph to the bloodstream can amplify the host systemic inflammatory response $[9,10,19]$. Previous studies demonstrated that the translocated bacteria could remain in the MLN for up to seven days, indicating a prolonged period of GALT stimulation [19]. This long period of the bacteria remaining at the MLN can be related to the participation of BT in the exacerbation of the inflammatory response in the early phase of sepsis $[12,17]$. Accordingly, an experimental study demonstrated that the BT induction in animals with non-lethal sepsis model unleashed $50 \%$ of mortality [19], suggesting a causal relation of BT in the worsening of sepsis. Several studies also suggested the involvement of BT event associated with the origin or perpetuation of MODS by GALT activation and exacerbation of the inflammatory response $[7,9,10,14,19]$, and others referred to the harmful synergistic inflammatory effect of BT in sepsis and critical illnesses [6-8-10,14,17,18,29].

\section{Conclusion}

According to the current concept, the gastrointestinal tract acts as a defense against invasion of intraluminal microorganisms, first as a biological barrier, which goes through continuous improvement of the gut immune barrier through physiological transloca- 
tion of selected microorganisms, and second by its mechanical barrier composed by a complex tissue organization [26,30].

The global results found in this experimental study using an acute animal model of sepsis showed BT as an important factor to mortality, and dependent on the bacterial strain that promotes sepsis. However, that is not known if BT is restricted to the acute phase of sepsis or occurs throughout the entire course of the disease, a question that needs to be elucidated.

The importance of BT in worsening human sepsis needs to be better elucidated. The concern with BT in critical diseases patients has resulted in therapeutic procedures like selective intestinal decontamination and the fecal microbiota transplantation [31]. These approaches have been applied as an attempt of improving critically ill patients since in clinical practice it is not possible to diagnose the BT occurrence, mainly due to ethical issues that preclude invasive processes in impaired patients.

The experimental findings of the present study suggest the need for more studies of the microbiota associated with sepsis and the importance of the diagnostic of intestinal microbiota imbalance to develop methods in the prevention of pathological BT. The feces microbiological culture to evaluate the overgrowth of the aerobic and the facultative anaerobic bacteria is a more accessible method than molecular techniques and can assist the laboratories of the developing countries in detecting intestinal dysbiosis and suspect the ongoing BT.

\section{Acknowledgment}

Supported by Fundação de Amparo à Pesquisa do Estado de São Paulo (FAPESP), Process number: 2017/21052-0

\section{Conflicts of Interest}

The author declares no financial conflicts of interest.

\section{Bibliography}

1. Singer M., et al. "The Third International Consensus Definitions for Sepsis and Septic Shock (Sepsis-3)". Journal of the American Medical Association 23.8 (2016): 801-810.

2. Taniguchi LU., et al. "Sepsis-related deaths in Brazil: an analysis of the national mortality registry from 2002 to 2010 ". Critical Care 18 (2014): 608.
3. Mayr FB., et al. "Epidemiology of severe sepsis". Virulence 5.1 (2014): 4-11.

4. Miller SI., et al. "LPS, TLR4 and infectious disease diversity". Nature Reviews Microbiology 3 (2005): 36-46.

5. Akira S., et al. "Pathogen recognition and innate immunity". Cell 124 (2006): 783- 801.

6. MacFie J., et al. "Gut origin of sepsis: a prospective study investigating associations between bacterial translocation, gastric microflora, and septic morbidity". Gut 45 (1999): 223-228.

7. Clark JA and Coopersmith CM. "Intestinal crosstalk: a new paradigm for understanding the gut as the "motor" of critical illness". Shock 28 (2007): 384-393.

8. Carrico CJ., et al. "Multiple-organ-failure syndrome. The gastrointestinal tract: the"motor" of MOF". The Archives of Surgery 121 (1986): 196-208.

9. Mainous MR., et al. "The gut: a cytokine-generating organ in sysemic inflammation?" Shock 4 (1995):193-199.

10. Deitch EA., et al. "Intestinal bacterial overgrowth induces the production of biologically active intestinal lymph". Journal of Trauma 56 (2004): 105-110.

11. Krentz $\mathrm{T}$ and Allen S. "Bacterial translocation in critical illness". Journal of Small Animal Practice 58.4 (2017):191-198.

12. Berg RD and Garlington AW. "Translocation of certain indigenous bacteria from the gastrointestinal tract to the mesenteric lymph nodes and other organs in a gnotobiotic mouse model". Infection and Immunity 23 (1979): 403-411.

13. Berg RD. "Bacterial translocation from the gastrointestinal tract”. Journal of Medicinal Chemistry 23 (1992): 217-244.

14. Deitch EA., et al. "Role of the gut in the development of injury and shock induced SIRS and MODS: the gut-lymph hypothesis, a review". Frontiers in Bioscience 11 (2006): 520-528.

15. Leaphart CL and Tepas JJ. "The gut is a motor of organ system dysfunction". Surgery 141 (2007): 563-569.

16. O'Boyle CJ., et al. "Microbiology of bacterial translocation in humans". Gut 42 (1998): 29-35.

17. Badami CD., et al. "Mesenteric lymph duct ligation improves survival In a lethal shock model". Shock 30 (2008): 680-685. 
18. Deitch EA., et al. "A time course study of the protective effect of mesenteric lymph duct ligation on hemorrhagic shock-induced pulmonary injury and the toxic effects of lymph from shocked rates on endothelial cell monolayer permeability". Surgery 129 (2001): 39-47.

19. Koh IHJ., et al. "Bacterial translocation, microcirculation injury and sepsis". Endocrine, Metabolic and Immune Disorders - Drug Targets 6 (2006): 7-16.

20. Koh IHJ., et al. "Microcirculatory evaluation in sepsis: a difficult task". Shock 34 (2010): 27-33.

21. Legrand M., et al. "The response of the host microcirculation to bacterial sepsis: does the pathogen matter?" Journal of Molecular Medicine 88.2 (2010): 127-133.

22. Da Silva., et al. "Uropathogenic Escherichia coli pathogenicity islands and other ExPEC virulence genes may contribute to the genome variability of enteroinvasive E. coli". BMC Microbiology 17 (2017): 1-8.

23. Guarner F. "Enteric flora in health and disease". Digestion 73.1 (2006): 5-12.

24. Shimizu K., et al. "Altered Gut Flora and Environment in Patients with Severe SIRS". The Journal of Trauma 60 (2006): $126-133$.

25. Steffen EK., et al. "Comparison of translocation rates of various indigenous bacteria from the gastrointestinal tract to the mesenteric lymph node". The Journal of Infectious Diseases 157 (1988): 1032-1038.

26. Assimakopoulosa SF., et al. "The Role of the Gut Barrier Function in Health and Disease". Gastroenterology Research 11.4 (2018): 261-263.

27. Hinshaw LB. "Sepsis/septic shock: Participation of the microcirculation: An abbreviated review". Critical Care Medicine 24.6 (1996): 1072-1078.

28. Fink MP. "Intestinal epithelial hyperpermeability: update on the pathogenesis of gut mucosal barrier dysfunction in critical illness". Current Opinion in Critical Care 9 (2003): 143-151.

29. MacFie J., et al. "Bacterial translocation studied in 927 patients over 13 years". British Journal of Surgery 93 (2006): 87-93.

30. Brenchley JM and Douek DC. "Microbial Translocation Across the GI Tract". Annual Review of Immunology 30 (2012):149173.
31. Gupta S., et al. "Fecal microbiota transplantation: in perspective". Therapeutic Advances in Gastroenterology 9.2 (2016): 229-239.

\section{Assets from publication with us}

- Prompt Acknowledgement after receiving the article

- Thorough Double blinded peer review

- Rapid Publication

- Issue of Publication Certificate

- High visibility of your Published work

Website: https://www.actascientific.com/

Submit Article: https://www.actascientific.com/submission.php Email us: editor@actascientific.com

Contact us: +919182824667 